\title{
CORRELATED CONTINUOUS TIME RANDOM WALKS
}

\author{
MARK M. MEERSCHAERT, ERKAN NANE, AND YIMIN XIAO
}

\begin{abstract}
Continuous time random walks impose a random waiting time before each particle jump. Scaling limits of heavy tailed continuous time random walks are governed by fractional evolution equations. Space-fractional derivatives describe heavy tailed jumps, and the time-fractional version codes heavy tailed waiting times. This paper develops scaling limits and governing equations in the case of correlated jumps. For long-range dependent jumps, this leads to fractional Brownian motion or linear fractional stable motion, with the time parameter replaced by an inverse stable subordinator in the case of heavy tailed waiting times. These scaling limits provide an interesting class of non-Markovian, non-Gaussian self-similar processes.
\end{abstract}

\section{INTRODUCTION}

Continuous time random walks (CTRW) separate IID particle jumps $\left(Y_{n}\right)$ by IID waiting times $\left(J_{n}\right)$. CTRW models are important in applications to geology 77, physics [28], and finance [32]. In the case of heavy tailed waiting times, CTRW scaling limits are subordinated processes that are self-similar but non-Markovian [27]. Their transition densities are governed by fractional diffusion equations [25]. Fractional diffusion equations replace the usual integer order derivatives in the diffusion equation by their fractional-order analogues [29, 30]. Just as the diffusion equation $\partial_{t} u=a \partial_{x}^{2} u$ governs the scaling limit of a simple random walk, the fractional diffusion equation $\partial_{t}^{\beta} u=a \partial_{x}^{\alpha} u$ governs the scaling limit of a CTRW with heavy tail jumps $\mathbb{P}\left(Y_{n}>r\right) \sim$ $r^{-\alpha}$ for $0<\alpha<2$ and waiting times $\mathbb{P}\left(J_{n}>t\right) \sim t^{-\beta}$ for $0<\beta<1$.

This paper develops limit theorems and governing equations for CTRW with correlated jumps $Y_{n}=\sum_{j} c_{j} Z_{n-j}$, where $\left(Z_{n}\right)$ are IID and $\left(c_{n}\right)$ are real numbers (see Section 2 for precise conditions). These CTRW models are useful for correlated observations separated by random waiting times, which are common, for example, in finance [31. Scaling limits of the partial sum process $S(t)=Y_{1}+\cdots+Y_{[t]}$ in the case of long range dependence include fractional Brownian motion (FBM) for light-tailed jumps [16, 36], and linear fractional stable motion (LFSM) for heavytailed jumps [1, 21, 36]. Letting $T_{n}=J_{1}+\cdots+J_{n}$ the time of the $n$th jump, and $N_{t}=\max \left\{n: T_{n} \leq t\right\}$ the number of jumps by time $t>0$, the scaling limit of the

Key words and phrases. Fractional Brownian motion, Lévy process, strictly stable process, linear fractional stable motion, waiting time process, local time, self-similarity, scaling limit.

Research of M. M. Meerschart was partially supported by NSF grant DMS-0706440.

Research of Y. Xiao was partially supported by NSF grant DMS-0706728. 
CTRW $S\left(N_{t}\right)$ is a FBM or LFSM subordinated to an inverse stable subordinator, which is connected to the local time of a strictly stable Lévy process [26], or the supremum process of a spectrally negative stable Lévy process [11]. This extends the results of [27, 6] to the case of dependent jumps. We also discuss some interesting properties of these self-similar limit processes, and governing equations for their probability densities.

\section{Results}

Let $\left\{Z_{n},-\infty<n<\infty\right\}$ denote IID random variables that belong to the strict domain of attraction of some stable law $A$ with index $0<\alpha \leq 2$. This means that the sequence of partial sums $P(n)=Z_{1}+\cdots+Z_{n}$ satisfies $a_{n} P(n) \Rightarrow A$ for some $a_{n}>0$, see Feller [18, p.312-313] or Whitt [36, p.114-115].

The particle jumps that we consider in this paper are given by the stationary linear process $\left\{Y_{n},-\infty<n<\infty\right\}$ defined by $Y_{n}=\sum_{j=0}^{\infty} c_{j} Z_{n-j}$, where $c_{j}$ are real constants such that $\sum_{j=0}^{\infty}\left|c_{j}\right|^{\rho}<\infty$ for some $\rho \in(0, \alpha)$. This condition ensures that the the series $\sum_{j=0}^{\infty} c_{j} Z_{n-j}$ converges in $L^{\rho}(\mathbb{P})$ and almost surely (see Avram and Taqqu [2]).

Let $J_{n}>0$ be IID waiting times, $T_{n}=J_{1}+\cdots+J_{n}$ the time of the $n$th particle jump, and $N_{t}=\max \left\{n: T_{n} \leq t\right\}$ the number of jumps by time $t>0$. Let $S(n)=Y_{1}+\cdots+Y_{n}$ denote the location of the particle after $n$ jumps, so that the continuous time random walk (CTRW) $S\left(N_{t}\right)$ gives the location of the particle at time $t>0$. Suppose that $J_{n}$ belongs to the domain of attraction of some stable law $D$ with index $0<\beta<1$ and $D>0$ almost surely. Hence $b_{n} T_{n} \Rightarrow D$ for some norming constants $b_{n}>0$. Let $b(t)=b_{[t]}$ and take $\tilde{b}(t)$ an asymptotic inverse of the regularly varying function $1 / b(t)$, so that $t b(\tilde{b}(t)) \rightarrow 1$ as $t \rightarrow \infty[27$.

Let $\{A(t), t \geq 0\}$ and $\{D(t), t \geq 0\}$ be stable Lévy processes with $A(1)=A, D(1)=$ $D$, respectively. Note that $\{D(t), t \geq 0\}$ is a stable subordinator of index $\beta$, hence its sample functions are almost surely strictly increasing [8, p.75]. Therefore, the inverse or hitting time process of $\{D(t), t \geq 0\}$,

$$
E_{t}=\inf \{x>0: D(x)>t\}, \quad \forall t \geq 0
$$

is well defined and the function $t \mapsto E_{t}$ is strictly increasing almost surely.

Our first result shows that the CTRW scaling limit in the case of short-range dependence is quite similar to the case of independent jumps studied by Meerschaert and Scheffler [27.

Theorem 2.1. Under the conditions of this section, suppose that $0<\alpha<2, c_{j} \geq 0$ and $\sum_{j} c_{j}^{\rho}<\infty$ for some $\rho \in(0, \alpha)$ with $\rho \leq 1$, and that one of the following holds:

(a) $0<\alpha \leq 1$; or

(b) $c_{j}=0$ for all but finitely many $j$; or

(c) $1<\alpha<2, c_{j}$ is monotone and $\sum_{j} c_{j}^{\rho}<\infty$ for some $\rho<1$. 
Then we have

$$
w^{-1} a_{[\tilde{b}(c)]} S\left(N_{c t}\right) \Rightarrow A\left(E_{t}\right)
$$

as $c \rightarrow \infty$ in the $M_{1}$ topology on $D([0, \infty), \mathbb{R})$, where $w=\sum_{j} c_{j}$.

In view of Avram and Taqqu [2], the convergence in (2.1) cannot be strengthened to the $J_{1}$ topology. Note that the processes $\{A(t), t \geq 0\}$ and $\left\{E_{t}, t \geq 0\right\}$ are self-similar, that is, for every constant $c>0$

$$
\{A(c t), t \geq 0\} \stackrel{d}{=}\left\{c^{1 / \alpha} A(t), t \geq 0\right\}
$$

and

$$
\left\{E_{c t}, t \geq 0\right\} \stackrel{d}{=}\left\{c^{\beta} E_{t}, t \geq 0\right\}
$$

where $\stackrel{d}{=}$ means equality in all finite dimensional distributions. It follows immediately that the scaling limit $\left\{A\left(E_{t}\right), t \geq 0\right\}$ in (2.1) is self-similar with index $\beta / \alpha$. When $0<\beta \leq 1 / 2$, the inner process $E_{t}$ in (2.1) is also the local time at zero of a strictly stable Lévy motion [26]. When $1 / 2 \leq \beta<1$, the inner process $E_{t}$ is also the supremum process of a stable Lévy motion with index $1 / \beta$ and no negative jumps [11].

Let $\partial_{t}^{\beta} g(t)$ denote the Caputo fractional derivative, the inverse Laplace transform of $s^{\beta} \tilde{g}(s)-s^{\beta-1} g(0)$ where $\tilde{g}(s)=\int_{0}^{\infty} e^{-s t} g(t) d t$ is the usual Laplace transform of $g$. Let $\partial_{ \pm x}^{\alpha} f(x)$ denote the Liouville fractional derivative, the inverse Fourier transform of $( \pm i k)^{\alpha} \hat{f}(k)$, where $\hat{f}(k)=\int_{-\infty}^{\infty} e^{-i k x} f(x) d s$ is the usual Fourier transform. The stable random variable $A(t)$ has a smooth density with Fourier transform $e^{-t \psi(k)}$ where $\psi(k)=a\left[p(i k)^{\alpha}+q(-i k)^{\alpha}\right]$ with $0 \leq p, q \leq 1$ and $p+q=1$ [24]. Then the limit $A\left(E_{t}\right)$ in (2.1) has a density $h(x, t)$ that solves the fractional diffusion equation $\partial_{t}^{\beta} h=a p \partial_{x}^{\alpha} h+a q \partial_{-x}^{\alpha} h$, see [27].

Next we consider the CTRW scaling limit for heavy-tailed particle jumps with long-range dependence. To simplify the presentation, we assume $a_{n}=n^{-1 / \alpha}$ (domain of normal attraction) and power-law weights; namely $c_{j} \sim c_{0} j^{H-1-1 / \alpha}$ as $j \rightarrow \infty$, for some $c_{0}>0$. The case $0<H<1 / \alpha$ means the stationary sequence $\left\{Y_{n}\right\}$ has short-range dependence, while the case $1 / \alpha<H<1$ means $\left\{Y_{n}\right\}$ has longrange dependence. The scaling limit of CTRW with short-range dependence has been partially covered by Theorem 2.1. The rest of the cases are treated in Theorems 2.2 and 2.3 below.

We will make use of the following definition. Given constants $\alpha \in(0,2)$ and $H \in(0,1)$, the $\alpha$-stable process $\left\{L_{\alpha, H}(t), t \in \mathbb{R}\right\}$ defined by

$$
L_{\alpha, H}(t)=\int_{\mathbb{R}}\left[(t-s)_{+}^{H-1 / \alpha}-(-s)_{+}^{H-1 / \alpha}\right] A(d s)
$$

is called a linear fractional stable motion (LFSM) with indices $\alpha$ and $H$. In the above, $a_{+}=\max \{0, a\}$ for all $a \in \mathbb{R}$ and $\{A(t), t \in \mathbb{R}\}$ is a two-sided strictly stable Lévy process of index $\alpha$ with $A(1)=A$ given at the beginning of Section 2. Namely, 
$n^{-1 / \alpha} P(n) \Rightarrow A$ as $n \rightarrow \infty$. Because of this, $\left\{L_{\alpha, H}(t), t \in \mathbb{R}\right\}$ defined by $(2.2)$ differs from the LFSM in Theorem 4.7.2 in [36] by a constant factor. Note that, when $H=1 / \alpha, L_{\alpha, H}(t)=A(t)$ for all $t \geq 0$. When $H \neq 1 / \alpha$, the stochastic integral in (2.2) is well-defined because

$$
\int_{\mathbb{R}}\left|(t-s)_{+}^{H-1 / \alpha}-(-s)_{+}^{H-1 / \alpha}\right|^{\alpha} d r<\infty
$$

See [33, Chapter 3].

By (2.2), it can be verified that $\left\{L_{\alpha, H}(t), t \in \mathbb{R}\right\}$ is $H$-self-similar with stationary increments [33, Proposition 7.4.2]. It is an $\alpha$-stable analogue of fractional Brownian motion and its probabilistic and statistical properties have been investigated by many authors. In particular, it is known that

(i) If $1 / \alpha<H<1$ (this is possible only when $1<\alpha<2$ ), then the sample function of $\left\{L_{\alpha, H}(t), t \in \mathbb{R}\right\}$ is almost surely continuous.

(ii) If $0<H<1 / \alpha$, then the the sample function of $\left\{L_{\alpha, H}(t), t \in \mathbb{R}\right\}$ is almost surely unbounded on every interval of positive length.

We refer to [33, Chapters 10 and 12] for more information.

Theorem 2.2. We assume the setting of this section. If $1<\alpha<2,1 / \alpha<H<1$, and $c_{j} \sim c_{0} j^{H-1-1 / \alpha}$ as $j \rightarrow \infty$ for some $c_{0}>0$, then as $c \rightarrow \infty$

$$
[\tilde{b}(c)]^{-H} S\left(N_{c t}\right) \Rightarrow K_{1} L_{\alpha, H}\left(E_{t}\right)
$$

in the $J_{1}$ topology on $D([0, \infty), \mathbb{R})$, where $K_{1}=c_{0} \alpha /(H \alpha-1)$.

The topology on $D([0, \infty), \mathbb{R})$ in Theorem 2.2 is stronger than that in Theorem 2.1, thanks to the fact that $L_{\alpha, H}(t)$ is a.s. continuous whenever $1 / \alpha<H<1$.

Observe that the case when $0<H<1 / \alpha$ and the constants $c_{j}(j \geq 0)$ are not all nonnegative is left uncovered by Theorems 2.1 and 2.2 . Because of Property (ii) of $\left\{L_{\alpha, H}(t), t \in \mathbb{R}\right\}$, the limiting process does not belong to the function space $D([0, \infty), \mathbb{R})$. Nevertheless, we have the following theorem.

Theorem 2.3. We assume the setting of this section. If $0<\alpha<2,0<H<1 / \alpha$, $c_{j} \sim c_{0} j^{H-1-1 / \alpha}$ as $j \rightarrow \infty$ for some $c_{0}>0$, and $\sum_{j} c_{j}=0$, then

$$
[\tilde{b}(c)]^{-H} S\left(N_{c t}\right) \stackrel{f . d .}{\longrightarrow} K_{1} L_{\alpha, H}\left(E_{t}\right)
$$

as $c \rightarrow \infty$, where $\stackrel{f . d .}{\longrightarrow}$ means convergence of all finite-dimensional distributions and $K_{1}=c_{0} \alpha /(H \alpha-1)$.

It is interesting to note that the constants in Theorems 2.2 and 2.3 are determined by $c_{0}, \alpha$ and $H$ in the same way. But $K_{1}$ is positive is when $1 / \alpha<H<1$, and is negative when $0<H<1 / \alpha$.

It follows from the self-similarity of $\left\{L_{\alpha, H}(t), t \in \mathbb{R}\right\}$ and $\{E(t), t \geq 0\}$ that the scaling limits in (2.3) and (2.4) are self-similar with index $H \beta$. When $1 / \alpha<H<1$, 
it can be seen that $\left\{L_{\alpha, H}\left(E_{t}\right), t \geq 0\right\}$ has continuous sample functions almost surely. However, if $0<H<1 / \alpha$, then $\left\{L_{\alpha, H}\left(E_{t}\right), t \geq 0\right\}$ is almost surely unbounded on every interval of positive length. It would be interesting to further study the properties of the process $\left\{L_{\alpha, H}\left(E_{t}\right), t \geq 0\right\}$.

We mention that both Theorems 2.2 and 2.3 can be extended to $\left(Z_{n}\right)$ in the strict domain of attraction of $A$ and $\left(c_{j}\right)$ regularly varying at $\infty$ with index $H-1-1 / \alpha$, using a slightly different normalization in (2.3) depending on $a_{n}$ and the probability tail of $Z_{n}$, compare [1].

Finally we consider the case $\alpha=2$. If $\{A(t), t \in \mathbb{R}\}$ in (2.2) is replaced by ordinary two-sided Brownian motion, then (2.2) defines a fractional Brownian motion $W_{H}=\left\{W_{H}(t), t \in \mathbb{R}\right\}$ on $\mathbb{R}$ of index $H$, which is a Gaussian process with mean zero and covariance function

$$
\mathbb{E}\left[W_{H}(t) W_{H}(s)\right]=\frac{1}{2}\left[|t|^{2 H}+|s|^{2 H}-|t-s|^{2 H}\right] .
$$

Theorem 2.4 gives the CTRW scaling limit for light-tailed particle jumps with long-range dependence.

Theorem 2.4. We assume the setting of this section. If $\alpha=2, \mathbb{E}\left[Z_{n}\right]=0, \mathbb{E}\left[Z_{n}^{2}\right]<$ $\infty, \sum_{j} c_{j}^{2}<\infty, \operatorname{Var}[S(n)]=\sigma_{n}^{2}$ varies regularly at $\infty$ with index $2 H$ for some $0<H<1$, and $\mathbb{E}\left[S(n)^{2 \rho}\right] \leq K_{2}\left[\mathbb{E}\left(S(n)^{2}\right)\right]^{\rho}$ for some constants $K_{2}>0$ and $\rho>1 / H$, then as $c \rightarrow \infty$

$$
\sigma_{[\tilde{b}(c)]}^{-1} S\left(N_{c t}\right) \Rightarrow W_{H}\left(E_{t}\right)
$$

in the $J_{1}$ topology on $D([0, \infty), \mathbb{R})$.

Note that it is not difficult to provide examples of sequences of IID random variables $\left\{Z_{n}\right\}$ and real numbers $\left\{c_{j}\right\}$ that satisfy the conditions of Theorem 2.4, see [16, 19]. It follows from the results of Taqqu [35] that the conclusion of Theorem 2.4 still holds if the linear process $\left\{Y_{n}\right\}$ is replaced by the stationary sequence $\left\{g\left(\xi_{n}\right)\right\}$, where $\left\{\xi_{n}\right\}$ is a stationary Gaussian sequence with mean 0, variance 1 and long-range dependence, and $g \in L^{2}\left(e^{-x^{2} / 2} d x\right)$ is a function with Hermite rank 1.

Theorem 2.4 contains the case $H=1 / 2$ where $W_{H}(t)=A(t)$ is a standard Brownian motion. This includes the situation of mean zero finite variance particle jumps, and heavy tailed waiting times between jumps. In this case, the CTRW scaling limit $A\left(E_{t}\right)$ has a density $h(x, t)$ that solves the time-fractional diffusion equation $\partial_{t}^{\beta} h=a \partial_{x}^{2} h$, see [27]. Since $\left\{W_{H}(c t), t \geq 0\right\} \stackrel{d}{=}\left\{c^{H} W_{H}(t), t \geq 0\right\}$, the scaling limit in (2.5) is selfsimilar with index $H \beta$. Some results on large deviation and sample path regularity have recently been obtained for $\left\{W_{H}\left(E_{t}\right), t \geq 0\right\}$ in [26].

In the case of finite mean waiting times, the CTRW scaling limit is essentially the same as for the underlying random walk. If $\mu=\mathbb{E} J_{n}<\infty$, then $\mu N_{t} / t \rightarrow 1$ almost surely as $t \rightarrow \infty$, and a simple argument along the lines of the proof of Theorem 2.1 
shows that $w^{-1} a_{[c]} S\left(N_{c t}\right) \Rightarrow A(t / \mu)$ in the $M_{1}$ topology on $D([0, \infty), \mathbb{R})$. Theorems $2.2,2.3$ and 2.4 can be extended similarly.

An easy argument with Fourier transforms shows that the density $h(x, t)$ of $L_{\alpha, H}(t)$ solves $\partial_{t} h=\alpha H t^{\alpha H-1}\left[a p \partial_{x}^{\alpha} h+a q \partial_{-x}^{\alpha} h\right]$. A similar argument shows that the density of $W_{H}(t)$ solves $\partial_{t} h=2 H t^{2 H-1} a \partial_{x}^{2} h$. An interesting open question is to establish the governing equation for the CTRW scaling limit in (2.3) and (2.5). This is not as simple as replacing the first time derivative by a fractional derivative in the governing equation for the outer process, since the $t$ variable also appears on the right-hand side, so that Theorem 3.1 of [3] does not apply.

\section{Proofs}

The proofs in this section are based on invariance principles for stationary sequences with short or long-range dependence (see, for example, [36]) and the CTRW limit theory developed in [27]. Due to the non-Markovian nature of the CTRW scaling limits in this paper, standard subordination methods can not be applied directly. Instead we apply continuous mapping-type arguments to prove Theorems $2.1,2.2$ and 2.4. The proof of Theorem 2.3 is quite different and relies on a criterion for the convergence of all finite-dimensional distributions of composite processes established by Becker-Kern, Meerschaert and Scheffler [6].

Recall that $J_{n}>0$ are IID waiting times, $T_{n}=J_{1}+\cdots+J_{n}$ the time of the $n$th particle jump, and $N_{t}=\max \left\{n: T_{n} \leq t\right\}$ the number of jumps by time $t>0$. Since $J_{n}$ belongs to the domain of attraction of some stable law $D$ with index $0<\beta<1$ and $D>0$ almost surely, with $b_{n} T_{n} \Rightarrow D$ for some norming constants $b_{n}>0$, the sequence $b_{n}$ varies regularly with index $-1 / \beta$, see [18]. Then the asymptotic inverse $\tilde{b}(t)$ of $1 / b$ varies regularly with index $\beta$, see Seneta [34]. Recall that the stable Lévy motion $\{D(x), x \geq 0\}$ with $D(1)=D$ is a stable subordinator of index $\beta$ and thus is strictly increasing [8, p.75]. Its inverse or hitting time process $E_{t}=\inf \{x>0: D(x)>t\}$ is strictly increasing with continuous sample paths, has moments of all orders, and its increments are neither stationary nor independent [27]. Bingham [10] shows that $E_{t}$ has a Mittag-Leffler distribution, and gives a differential equation that governs its finite dimensional distributions.

Proof of Theorem 2.1. Corollary 3.4 in [27] shows that $\tilde{b}(c)^{-1} N_{c t} \Rightarrow E_{t}$ as $c \rightarrow \infty$ in the $J_{1}$ topology on $D([0, \infty),[0, \infty))$. Note that $\tilde{b}(c) \rightarrow \infty$ as $c \rightarrow \infty$ since this function is regularly varying at $\infty$ with index $\beta>0$. Theorem 4.7.1 in Whitt [36] shows that $a_{n} S(n t) \Rightarrow w A(t)$ in the $M_{1}$ topology on $D([0, \infty), \mathbb{R})$. Since the $J_{1}$ topology is stronger, and since the waiting times $\left(J_{n}\right)$ are independent of $\left(Y_{n}\right)$, we have

$$
\left(a_{[\tilde{b}(c)]} S(\tilde{b}(c) t), \tilde{b}(c)^{-1} N_{c t}\right) \Rightarrow\left(A(t), E_{t}\right)
$$

in the $M_{1}$ topology of the product space $D([0, \infty), \mathbb{R} \times[0, \infty))$. Of course, this last statement also follows from Theorem 3.2 in [9]. 
Since the process $\left\{E_{t}, t \geq 0\right\}$ is strictly increasing and continuous, Theorem 13.2.4 in [36] yields

$$
a_{[\tilde{b}(c)]} S\left(\tilde{b}(c) \cdot \tilde{b}(c)^{-1} N_{c t}\right) \Rightarrow A(E(t))
$$

in the $M_{1}$ topology on $D([0, \infty), \mathbb{R})$, which completes the proof.

Proof of Theorem 2.2. Recall that $\tilde{b}(c)^{-1} N_{c t} \Rightarrow E_{t}$ in the $J_{1}$ topology on $D([0, \infty)$, $[0, \infty)$ ) [27, Corollary 3.4]. Theorem 4.7.2 in Whitt [36], originally due to Astrauskas [1], shows that $n^{-H} S(n t) \Rightarrow K_{1} L_{\alpha, H}(t)$ in the $J_{1}$ topology on $D([0, \infty), \mathbb{R})$, where $K_{1}=c_{0} \alpha /(H \alpha-1)$.

Since $\left\{N_{t}, t \geq 0\right\}$ is independent of $\{S(n), n \geq 1\}$, we have

$$
\left([\tilde{b}(c)]^{-H} S(\tilde{b}(c) t), \tilde{b}(c)^{-1} N_{c t}\right) \Rightarrow\left(K_{1} L_{\alpha, H}(t), E_{t}\right)
$$

in the product space. Combining this with [36, Theorem 13.2.4] yields (2.5) in the $M_{1}$ topology. Since both processes $\left\{L_{\alpha, H}(t), t \geq 0\right\}$ and $\left\{E_{t}, t \geq 0\right\}$ are continuous, and the latter is strictly increasing, one can apply Theorem 13.3.1 in [36] to strengthen to the conclusion to convergence in the $J_{1}$ topology. This proves Theorem 2.2 .

Proof of Theorem [2.3. It is sufficient to show that for all integers $m \geq 1,0<t_{1}<$ $\cdots<t_{m}$, we have

$$
\tilde{b}(c)^{-H}\left(S\left(N_{c t_{1}}\right), \ldots, S\left(N_{c t_{m}}\right)\right) \Rightarrow K_{1}\left(L_{\alpha, H}\left(E_{t_{1}}\right), \ldots, L_{\alpha, H}\left(E_{t_{m}}\right)\right)
$$

as $c \rightarrow \infty$. For this purpose, we will make use of Proposition 4.1 in [6], which provides a useful criterion for the convergence of all finite-dimensional distributions of composite processes, and Corollary 3.3 in [21] which is concerned with convergence of finite dimensional distributions of weighted partial sums of IID random variables.

We will adopt some notation from [6]. For $\mathbf{t}=\left(t_{1}, \ldots, t_{m}\right)$ and $c>0$, let $\rho_{c}:=\rho_{c}^{\mathbf{t}}$ be the distribution of $\tilde{b}(c)^{-1}\left(N_{c t_{1}}, \ldots, N_{c t_{m}}\right)$, and let $\rho:=\rho^{\mathbf{t}}$ be the distribution of $\left(E_{t_{1}}, \ldots, E_{t_{m}}\right)$. Since $\tilde{b}(c)^{-1} N_{c t} \Rightarrow E_{t}$ in the $J_{1}$ topology on $D([0, \infty),[0, \infty))[27$, Corollary 3.4], we have $\rho_{c} \Rightarrow \rho$ as $c \rightarrow \infty$.

It follows from the definition of $\left\{Y_{n}\right\}$ that, for every $x \geq 0, S(n x)$ can be rewritten as

$$
S(n x)=\sum_{j=-\infty}^{\infty}\left(\sum_{k=1-j}^{[n x]-j} \widetilde{c}_{k}\right) Z_{j}
$$

where $\widetilde{c}_{k}=c_{k}$ if $k \geq 0$ and $\widetilde{c}_{k}=0$ if $k<0$. Under the assumptions of Theorem 2.3, we have $\sum_{k=-\infty}^{\infty}\left|\widetilde{c}_{k}\right|<\infty, \sum_{k=-\infty}^{\infty} \widetilde{c}_{k}=0$ and

$$
\sum_{k=n}^{\infty} \widetilde{c}_{k} \sim c_{0} \sum_{k=n}^{\infty} k^{H-1 / \alpha-1} \sim-\frac{c_{0} \alpha}{H \alpha-1} n^{H-1 / \alpha}
$$


as $n \rightarrow \infty$. Thus, the conditions of Theorem 5.2 in [21] are satisfied with $\psi(n)=$ $n^{H-1 / \alpha}, a=-K_{1}$ (recall that $\left.K_{1}=c_{0} \alpha /(H \alpha-1)\right), b=0$ and $A=0$. It follows that

$$
n^{-H} S(n t) \stackrel{f . d .}{\longrightarrow} K_{1} L_{\alpha, H}(t) \quad \text { as } n \rightarrow \infty .
$$

For any $\mathbf{x}=\left(x_{1}, \ldots, x_{m}\right) \in \mathbb{R}_{+}^{m}$, let $\mu_{c}(\mathbf{x})$ be the distribution of $\tilde{b}(c)^{-H}\left(S\left(\tilde{b}(c) x_{1}\right)\right.$, $\left.\ldots, S\left(\tilde{b}(c) x_{m}\right)\right)$ and let $\nu(\mathbf{x})$ be the distribution of $K_{1}\left(L_{\alpha, H}\left(x_{1}\right), \ldots, L_{\alpha, H}\left(x_{m}\right)\right)$. Then for every $c>0$, the mapping $\mathbf{x} \mapsto \mu_{c}(\mathbf{x})$ is weakly measurable. Since the linear fractional stable motion $\left\{L_{\alpha, H}(t), t \geq 0\right\}$ is stochastically continuous, the mapping $\mathbf{x} \mapsto \nu(\mathbf{x})$ is weakly continuous. Moreover, it follows from (3.3) that, for every $\mathbf{x} \in \mathbb{R}_{+}^{m}$, $\mu_{c}(\mathbf{x}) \Rightarrow \nu(\mathbf{x})$ as $c \rightarrow \infty$.

As in [6] we apply a conditioning argument and the independence between the sequences $\left\{Y_{n}\right\}$ and $\left\{J_{n}\right\}$ to derive that the distribution of $\tilde{b}(c)^{-H}\left(S\left(N_{c t_{1}}\right), \ldots, S\left(N_{c t_{m}}\right)\right)$ can be written as $\int_{\mathbb{R}_{+}^{m}} \mu_{c}(\mathbf{x}) d \rho_{c}(\mathbf{x})$, which is a probability measure on $\mathbb{R}^{m}$. Similarly, the distribution of the random vector $K_{1}\left(L_{\alpha, H}\left(E_{t_{1}}\right), \ldots, L_{\alpha, H}\left(E_{t_{m}}\right)\right)$ can be written as $\int_{\mathbb{R}_{+}^{m}} \nu(\mathbf{x}) d \rho(\mathbf{x})$.

Therefore, (3.1) follows from Proposition 4.1 in [6] once we verify that, for every $\mathbf{x} \in \mathbb{R}_{+}^{m}, \mu_{c}\left(\mathbf{x}^{(c)}\right) \Rightarrow \nu(\mathbf{x})$ for every sequence $\left\{\mathbf{x}^{(c)}\right\} \subset \mathbb{R}_{+}^{m}$ that satisfies $\mathbf{x}^{(c)} \rightarrow \mathbf{x}$ as $c \rightarrow \infty$.

The last statement is equivalent to

$$
c^{-H}\left(S\left(c x_{1}^{(c)}\right), \ldots, S\left(c x_{m}^{(c)}\right)\right) \Rightarrow K_{1}\left(L_{\alpha, H}\left(x_{1}\right), \ldots, L_{\alpha, H}\left(x_{m}\right)\right)
$$

whenever $\mathbf{x}^{(c)} \rightarrow \mathbf{x}$ as $c \rightarrow \infty$. This is stronger than (3.3), where the fixed timeinstants $0<x_{1}<x_{2}<\cdots<x_{m}$ are replaced now by $x_{1}^{(c)}, \ldots, x_{m}^{(c)}$. Our proof of (3.4) is a modification of the proof of Theorem 5.2 in Kasahara and Maejima [21].

To this end, we define the step function $r \mapsto A_{c}(r)$ on $\mathbb{R}$ by

$$
A_{c}(r)= \begin{cases}c^{-1 / \alpha} \sum_{j=1}^{[c r]} Z_{j} & \text { if } r>0 \\ c^{-1 / \alpha} \sum_{j=[c r]}^{0} Z_{j} & \text { if } r \leq 0 .\end{cases}
$$

Then for any function $g$ on $\mathbb{R}$, as in [21, p.88], we define

$$
\int_{-\infty}^{\infty} g(r) d A_{c}(r)=\frac{1}{c^{1 / \alpha}} \sum_{j=-\infty}^{\infty} g\left(\frac{j}{c}\right) Z_{j} .
$$

By using (3.2), (3.5) and (3.6) we can rewrite $c^{-H} S(c x)(x>0$ and $c>0)$ as

$$
\begin{aligned}
c^{-H} S(c x) & =\frac{1}{c^{1 / \alpha}} \sum_{j=-\infty}^{\infty} \frac{1}{c^{H-1 / \alpha}}\left(\sum_{k=1-j}^{[c x]-j} \widetilde{c}_{k}\right) Z_{j} \\
& =\frac{1}{c^{1 / \alpha}} \int_{\mathbb{R}} g_{c}(x, r) d A_{c}(r),
\end{aligned}
$$


where the integrand $g_{c}(x, r)$ is given by

$$
\begin{aligned}
g_{c}(x, r) & =\frac{1}{c^{H-1 / \alpha}} \sum_{k=1-[c r]}^{[c x]-[c r]} \widetilde{c}_{k} \\
& =\frac{1}{c^{H-1 / \alpha}}\left(\sum_{k=-[c r]+1}^{\infty} \widetilde{c}_{k}-\sum_{k=[c x]-[c r]+1}^{\infty} \widetilde{c}_{k}\right) .
\end{aligned}
$$

In the above, we have used the fact that $\sum_{j=-\infty}^{\infty} \widetilde{c}_{j}=0$ to derive the second equality.

It follows from (3.7) that (3.4) can be rewritten as

$$
\left\{\int_{\mathbb{R}} g_{c}\left(x_{i}^{(c)}, r\right) d A_{c}(r)\right\}_{i=1}^{m} \Rightarrow\left\{K_{1} \int_{\mathbb{R}} g\left(x_{i}, r\right) d A(r)\right\}_{i=1}^{m},
$$

where $g(x, r)=(x-r)_{+}^{H-1 / \alpha}-(-r)_{+}^{H-1 / \alpha}$ is the function in (2.2).

Now let us fix $\mathbf{x}=\left(x_{1}, \ldots, x_{m}\right) \in \mathbb{R}_{+}^{m}$ and an arbitrary sequence $\left\{\mathbf{x}^{(c)}\right\} \subset \mathbb{R}_{+}^{m}$ that satisfies $\mathbf{x}^{(c)} \rightarrow \mathbf{x}$ as $c \rightarrow \infty$. By Corollary 3.3 in [21] (with $f_{n}^{i}(\cdot)$ being taken as $\left.g_{c}\left(x_{i}^{(c)}, \cdot\right)\right)$, the convergence in (3.9) will follow once we verify that for every $1 \leq i \leq m$ the following conditions are satisfied:

$(\mathrm{A} 1)^{\prime}$ for $d r$-almost every $r \in \mathbb{R}$,

$$
g_{c}\left(x_{i}^{(c)}, r_{c}\right) \longrightarrow K_{1} g\left(x_{i}, r\right)
$$

whenever $r_{c} \rightarrow r$ as $c \rightarrow \infty$.

$(\mathrm{A} 2)^{\prime}$ for every $T>0$, there exists a constant $\beta>\alpha$ such that

$$
\sup _{c \geq 1} \int_{|r| \leq T}\left|g_{c}\left(x_{i}^{(c)}, r\right)\right|^{\beta} d \rho_{c}(r)<\infty,
$$

where $\rho_{c}(r)=[c r] / c$, and

$(\mathrm{A} 3)^{\prime}$ there exists an $\varepsilon>0$ such that

$$
\lim _{T \rightarrow \infty} \limsup _{c \rightarrow \infty} \int_{|r|>T}\left\{\left|g_{c}\left(x_{i}^{(c)}, r\right)\right|^{\alpha-\varepsilon}+\left|g_{c}\left(x_{i}^{(c)}, r\right)\right|^{\alpha+\varepsilon}\right\} d \rho_{c}(r)=0 .
$$

For simplicity of notation, we will from now on omit the subscript $i$. To verify Condition $(\mathrm{A} 1)^{\prime}$, note that by the property of $\left\{c_{k}\right\}$, we have

$$
\lim _{c \rightarrow \infty} \frac{1}{c^{H-1 / \alpha}} \sum_{k=[c r]+1}^{\infty} \widetilde{c}_{k}= \begin{cases}-K_{1} r^{H-1 / \alpha} & \text { if } r>0 \\ 0 & \text { if } r \leq 0\end{cases}
$$

and the convergence is uniform in $r$ on every compact set in $\mathbb{R} \backslash\{0\}$. For any $x \in \mathbb{R}_{+}$ and $r \in \mathbb{R}$, we may distinguish three cases $r<0,0 \leq r \leq x$ and $r>x$. By applying (3.13) to (3.8) we derive that, as $c \rightarrow \infty, g_{c}(x, r) \rightarrow g(x, r)$ uniformly in $(x, r)$ on every compact set in $\left\{(x, r): x \in \mathbb{R}_{+}, r \in \mathbb{R} \backslash\{0, x\}\right\}$. This implies that $g_{c}\left(x^{(c)}, r_{c}\right) \rightarrow g(x, r)$ whenever $r \neq x$ and $r_{c} \rightarrow r$ as $c \rightarrow \infty$. Hence (A1)' is satisfied. 
To verify Condition $(\mathrm{A} 2)^{\prime}$, we take a constant $\beta>\alpha$ such that $\beta(H-1 / \alpha)>-1$ and consider the integral

$$
\int_{|r| \leq T}\left|\frac{1}{c^{H-1 / \alpha}} \sum_{k=[c x]-[c r]+1}^{\infty} \widetilde{c}_{k}\right|^{\beta} d \rho_{c}(r)=\sum_{|j| \leq c T} \frac{1}{c^{\beta(H-1 / \alpha)+1}}\left|\sum_{k=[c x]-j+1}^{\infty} \widetilde{c}_{k}\right|^{\beta}
$$

Let $N>1$ be a constant such that $\left|c_{k}\right| \leq 2 c_{0} k^{H-1 / \alpha-1}$ for all $k \geq N$. We split the summation in the right-hand side of (3.14) according to whether $[c x]-j \leq N$ or $[c x]-j>N$. Thanks to the fact that $\sum_{k} \widetilde{c}_{k}=0$ we have

$$
\sum_{|j| \leq c T,[c x]-j \leq N} \frac{1}{c^{\beta(H-1 / \alpha)+1}}\left|\sum_{k=[c x]-j+1}^{\infty} \widetilde{c}_{k}\right|^{\beta} \leq \frac{K_{3}}{c^{\beta(H-1 / \alpha)+1}}
$$

for some constant $K_{3}>0$. In the above we have also used the fact that there are at most $N+1$ non-zero terms in the summation in $j$.

On the other hand, we have

$$
\begin{aligned}
& \sum_{|j| \leq c T,[c x]-j>N} \frac{1}{c^{\beta(H-1 / \alpha)+1}}\left|\sum_{k=[c x]-j+1}^{\infty} \widetilde{c}_{k}\right|^{\beta} \\
& \quad \leq K_{4} \sum_{|j| \leq c T,[c x]-j>N} \frac{([x]-[j / c])^{\beta(H-1 / \alpha)}}{c} \\
& \leq K_{5} \int_{|r| \leq T}|x-r|^{\beta(H-1 / \alpha)} d r
\end{aligned}
$$

for some constants $K_{4}, K_{5}>0$ and the last integral is convergent because $\beta(H-$ $1 / \alpha)>-1$. Combining (3.15) and (3.16) yields

$$
\begin{aligned}
& \int_{|r| \leq T}\left|\frac{1}{c^{H-1 / \alpha}} \sum_{k=[c x]-[c r]+1}^{\infty} \widetilde{c}_{k}\right|^{\beta} d \rho_{c}(r) \\
& \leq \frac{K_{3}}{c^{\beta(H-1 / \alpha)+1}}+K_{5} \int_{|r| \leq T}|x-r|^{\beta(H-1 / \alpha)} d r .
\end{aligned}
$$

Since the first sum in (3.8) corresponds to $x=0$, we see that (A2) follows from (3.17).

The verification of $(\mathrm{A} 3)^{\prime}$ is similar to the above and hence is omitted. This finishes the proof of Theorem 2.3 .

Finally we prove Theorem 2.4

Proof of Theorem 2.4. Recall that $\tilde{b}(c)^{-1} N_{c t} \Rightarrow E_{t}$ in the $J_{1}$ topology [27, Corollary 3.4]. Theorem 4.6.1 in Whitt [36] shows that, as $n \rightarrow \infty, \sigma_{n}^{-1} S(n t) \Rightarrow W_{H}(t)$ in the $J_{1}$ topology on $D([0, \infty), \mathbb{R})$. This result is originally due to Davydov [16], see also Giriatis et al. [19, p. 276]. Since the sequence $\left(J_{n}\right)$ is independent of $\left(Y_{n}\right)$, we have 
$\left(\sigma_{[\tilde{b}(c)]}^{-1} S(\tilde{b}(c) t), \tilde{b}(c)^{-1} N_{c t}\right) \Rightarrow\left(W_{H}(t), E_{t}\right)$ in the product space, and then continuous mapping along with Theorem 13.3.1 in [36] yields (2.5]) in the $J_{1}$ topology.

\section{Discussion}

Self-similar processes arise naturally in limit theorems of random walks and other stochastic processes, and they have been applied to model various phenomena in a wide range of scientific areas including telecommunications, turbulence, image processing and finance [17]. The most prominent example is fractional Brownian motion (FBM). However, many real data sets are non-Gaussian, which motivates the development of alternative models. Many authors have constructed and investigated various classes of non-Gaussian self-similar processes. Samorodnitsky and Taqqu [33] provide a systematic account on self-similar stable processes with stationary increments. Burdzy [12, 13] introduced iterated Brownian motion (IBM) which replaces the time parameter of a two-sided Brownian motion by an independent one-dimensional Brownian motion $B=\left\{B_{t}, t \geq 0\right\}$. In this paper we have shown that the limit processes of CTRWs with correlated jumps form a wide class of self-similar processes which are different from the existing ones.

When $0<\beta \leq 1 / 2$, the inner process $E_{t}$ in (2.1) or (2.5) is also the local time at zero $L_{t}$ of a stable Lévy process, and the iterated process $\left\{W_{H}\left(L_{t}\right), t \geq 0\right\}$ is called a local time fractional Brownian motion (LTFBM) in [26], a self-similar process with index $\beta H$ and continuous sample paths. Large deviation and modulus of continuity results for LTFBM are developed in a companion paper [26]. Strassen-type law of the iterated logarithm has been proved by Csáki, Földes and Révész [15] for local time Brownian motion (LTBM, the case $H=1 / 2$ ). It is interesting to note that our Theorem 2.4 shows that "randomly-stopped stationary sequence" $\left\{\left(Y_{n}: n \leq N_{t}\right), t \geq 0\right\}$ belongs to the domain of attraction of $\left\{W_{H}\left(L_{t}\right), t \geq 0\right\}$ for all $H \in(0,1)$. This theorem provides a physical interpretation of the process $\left\{W_{H}\left(L_{t}\right), t \geq 0\right\}$.

One interesting property of LTBM is that its increments are uncorrelated (this follows by simple conditioning argument), but not independent. It has long been recognized that price returns are essentially uncorrelated, but not independent [5, 23]. Hence LTBM, the scaling limit of a CTRW with (weakly) correlated price jumps, may be useful to model financial price returns. This approach could provide an interesting alternative to the subordinated variance-Gamma model of Madan and Seneta [22, 14] or the FATGBM model of Heyde [20].

LTBM has a close connection to fractional partial differential equations. Meerschaert and Scheffler [27] and Baeumer and Meerschaert [3] showed that the probability density $u(x, t)$ of LTBM solves the fractional Cauchy problem.

$$
\partial_{t}^{\beta} u(t, x)=\partial_{x}^{2} u(t, x) .
$$


Baeumer, Meerschaert and Nane [4] further showed that the density of the iterated Brownian motion solves the same equation (4.1). As we mentioned at the end of Section 2, the connection between the limit processes in this paper and fractional partial differential equations remains to be investigated.

\section{REFERENCES}

[1] A. Astrauskas, Limit theorems for sums of linearly generated random variables. Lithuanian Math. J. 23 (1983), 127-134.

[2] F. Avram and M. S. Taqqu, Weak convergence of sums of moving averages in the $\alpha$-stable domain of attraction. Ann. Probab. 20 (1992), 483-503.

[3] B. Baeumer and M. M. Meerschaert, Stochastic solutions for fractional Cauchy problems. Fractional Calculus Appl. Anal. 4 (2001), 481-500.

[4] B. Baeumer, M. M. Meerschaert and E. Nane, Brownian subordinators and fractional Cauchy problems. (2007), Trans. Amer. Math. Soc. (to appear).

[5] R. T. Baillie, T. Bollerslev and H. O. Mikkelsen, Fractionally integrated generalized autoregressive conditional heteroskedasticity. J. Econometrics 74 (1996), 3-30.

[6] P. Becker-Kern, M. M. Meerschaert and H. P. Scheffler, Limit theorems for continuous-time random walks with two scales. J. Appl. Probab. 41 (2004), 455-466.

[7] B. Berkowitz, A. Cortis, M. Dentz and H. Scher, Modeling non-Fickian transport in geological formations as a continuous time random walk. Rev. Geophys. 44 (2006), RG2003, doi:10.1029/2005RG000178.

[8] J. Bertoin, Lévy Processes. Cambridge University Press, 1996.

[9] P. Billingsley, Convergence of Probability Measures. John Wiley, New York, 1968.

[10] N. H. Bingham, Limit theorems for occupation times of Markov processes. Z. Wahrsch. verw Gebiete 17 (1971), 1-22.

[11] N. H. Bingham, Maxima of sums of random variables and suprema of stable processes. $Z$. Wahrsch. verw Gebiete 26 (1973), 273-296.

[12] K. Burdzy, Some path properties of iterated Brownian motion. In: Seminar on Stochastic Processes (E. Çinlar, K.L. Chung and M.J. Sharpe, eds.), pp. 67-87, Birkhäuser, Boston, 1993.

[13] K. Burdzy, Variation of iterated Brownian motion. In: Workshops and Conference on Measurevalued Processes, Stochastic Partial Differential Equations and Interacting Particle Systems (D.A. Dawson, ed.), pp. 35-53, Amer. Math. Soc. Providence, RI, 1994.

[14] P. Carr, H. Geman, D. Madan and M. Yor, The fine structure of asset returns: An empirical investigation. J. Business 75 (2002), 305-332.

[15] E. Csáki, A. Földes and P. Révész, Strassen theorems for a class of iterated processes. Trans. Amer. Math. Soc. 349 (1997), 1153-1167.

[16] Y. Davydov, The invariance principle for stationary processes. Teor. Verojatnost. i Primenen. 15 (1970), 498-509.

[17] P. Embrechts and M. Maejima, Selfsimilar Processes. Princeton University Press, Princeton, NJ, 2002.

[18] W. Feller, An Introduction to Probability Theory and Its Applications, Vol. II. 2nd Ed., Wiley, New York, 1971.

[19] L. Giraitisa, P. Kokoszka, L. Remigijus and G. Teyssière, Rescaled variance and related tests for long memory in volatility and levels. J. Econometrics 112 (2003), 265-294.

[20] C. C. Heyde, On modes of long-range dependence. J. Appl. Probab. 39 (2002), 882-888. 
[21] Y. Kasahara and M. Maejima, Weighted sums of I.I.D random variables attracted to integrals of stable processes. Probab. Th. Rel. Fields 78 (1988), 75-96.

[22] D. B. Madan and E. Seneta, The variance gamma (VG) model for share market returns. $J$. Business 63 (1990), 511-524.

[23] B. B. Mandelbrot (1963) The variation of certain speculative prices. J. Business 36, 394-419.

[24] M. M. Meerschaert and H. P. Scheffler, Limit Distributions for Sums of Independent Random Vectors: Heavy Tails in Theory and Practice. Wiley Interscience, New York, 2001.

[25] M. M. Meerschaert, D. A. Benson, H. P. Scheffler and B. Baeumer, Stochastic solution of space-time fractional diffusion equations. Phys. Rev. E 65 (2002), 1103-1106.

[26] M. M. Meerschaert, E. Nane and Y. Xiao, Large deviations for local time fractional Brownian motion and applications. J. Math. Anal. Appl. 346 (2008), 432-445.

[27] M. M. Meerschaert and H. P. Scheffler, Limit theorems for continuous time random walks with infinite mean waiting times. J. Appl. Probab. 41 (2004), 623-638.

[28] R. Metzler and J. Klafter, The restaurant at the end of the random walk: recent developments in the description of anomalous transport by fractional dynamics. J. Physics A 37 (2004), R161-R208.

[29] K. Miller and B. Ross, An Introduction to the Fractional Calculus and Fractional Differential Equations. Wiley and Sons, New York, 1993.

[30] S. Samko, A. Kilbas and O. Marichev, Fractional Integrals and Derivatives: Theory and Applications. Gordon and Breach, London, 1993.

[31] E. Scalas, R. Gorenflo and F. Mainardi, Fractional calculus and continuous-time finance. Phys. A 284 (2000), 376-384.

[32] E. Scalas, Five years of continuous-time random walks in econophysics. In: Proceedings of WEHIA 2004, A. Namatame (ed.), Kyoto, 2004.

[33] G. Samorodnitsky and M. S. Taqqu, Stable Non-Gaussian Processes. Chapman and Hall, 1994.

[34] E. Seneta, Regularly Varying Functions. Lecture Notes Math. 508, Springer, Berlin, 1976.

[35] M. S. Taqqu, Weak Convergence to fractional Brownian motion and to the Rosenblatt process. Z. Wahrsch. Verw. Gebiete 31 (1975), 287-302.

[36] W. Whitt, Stochastic-Process Limits. Springer, New York, 2002.

Mark M. Meerschaert, Department of Probability and Statistics, Michigan State UNIVERSity, EAST LANSING, MI 48823

E-mail address: mcubed@stt.msu.edu

URL: http://www.stt.msu.edu/ mcubed/

Erkan Nane, Department of Mathematics and Statistics, 221 Parker Hall, Auburn

University, Auburn, AL 36849

E-mail address: nane@stt.msu.edu

$U R L:$ http://www.stt.msu.edu/ nane

Yimin Xiao, Department Statistics and Probability, Michigan State University, EAST LANSING, MI 48823

E-mail address: xiao@stt.msu.edu

URL: http://www.stt.msu.edu/ xiaoyimi 